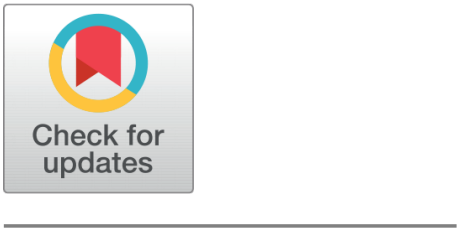

OPEN ACCESS

Received: $25-07-2020$

Accepted: $30-07-2020$

Published: 11-08-2020

Editor: Dr. Natarajan Gajendran

Citation: Saini S, Sharma S, Khangembam M, Singh V (2020) Structural analysis of the chemical vapour deposition grown molybdenum disulphide nanofilms for multifaceted applications. Indian Journal of Science and Technology 13(29): 2973-2980. https://doi.org/ 10.17485/IJST/v13i29.1215

*Corresponding author.

vinodsingh@dtu.ac.in, vinodsingh.iitd12@gmail.com

Funding: None

Competing Interests: None

Copyright: ( 2020 Saini et al. This is an open access article distributed under the terms of the Creative Commons Attribution License, which permits unrestricted use, distribution, and reproduction in any medium, provided the original author and source are credited.

Published By Indian Society for Education and Environment (iSee)

ISSN

Print: 0974-6846

Electronic: 0974-5645

\section{Structural analysis of the chemical vapour deposition grown molybdenum disulphide nanofilms for multifaceted applications}

\author{
Sonakshi Saini ${ }^{1}$, Sakshi Sharma ${ }^{1}$, Maya Khangembam ${ }^{1}$, Vinod Singh ${ }^{1 *}$ \\ 1 Department of Applied Physics, Delhi Technological University, Delhi, 110042, India
}

\section{Abstract}

Background/Objectives: In recent years, the research on molybdenum disulphide $\left(\mathrm{MoS}_{2}\right)$ has gained significance because of its unique properties and ease of incorporation in hybrid structures, which makes it one of the most suitable materials for devices and multifaceted Applications. The objective of the study is to synthesize $\mathrm{MoS}_{2}$ nanofilms and then to characterize them through X-ray diffraction (XRD) technique. Methods: In this study, $\mathrm{MoS}_{2}$ nanofilms are synthesized on silicon dioxide substrates by the thermal Chemical Vapour Deposition (CVD) technique, where molybdenum trioxide $\left(\mathrm{MoO}_{3}\right)(\mathrm{VI})$ powder and sulphur (S) flakes are used as precursors. Findings: $X$-ray diffraction (XRD) measurements have been carried out for the thermal CVD grown $\mathrm{MoS}_{2}$ nanofilm samples. Further, the observed XRD data has been analyzed and the structural analysis of synthesized $M_{0} S_{2}$ nanofilms is presented in this report. Furthermore, the experimentally observed findings are compared with the standard findings and shown that they are resembling closely. Novelty/Applications: In order to highlight the scope of our work, the important applications, of the molybdenum disulphide nanostructures are also discussed, that make $\mathrm{MoS}_{2}$ nanostructures attractive candidates in fields as diverse as energy, environmental, biomedical and semiconductors.

Keywords: Chemical vapour deposition; molybdenum disulphide; nanofilms; XRD; transition metal dichalcogenide

\section{Introduction}

Molybdenum disulphide $\left(\mathrm{MoS}_{2}\right)$ is a transition metal dichalcogenide that has gained widespread popularity among nanomaterials owing to its extraordinary properties ${ }^{(1-5)}$ and resulting applications in fields ranging from memory devices ${ }^{(6,7)}$, environmental ${ }^{(8)}$ and biomedical applications ${ }^{(9)}$ to optoelectronics ${ }^{(10)}$ and transistors ${ }^{(11)}$.

The need for smaller, more efficient electronic devices has made two dimensional (2-D) materials a focal point of research in materials science. Graphene, the most prominent member in the family of 2-D materials, has been a good candidate for nanoelectronics owing to its exceptional electronic behaviour - a characteristic quantum Hall effect, high thermal conductivity, high electron mobility, superior elastic strength 
and optical performance, and exploitable thermal stability. However, it is still far from being an ideal material for small scale transistors, owing to the absence of an electronic band gap ${ }^{(12-22)}$. Efforts focused on finding an alternate material has led to the enhanced prominence of a whole new variety of 2-D materials, among which transition metal dichalcogenides (TMDCs) such as molybdenum disulphide have been quite popular.

Molybdenum disulphide belongs to the family of transition metal dichalcogenides, materials having the generalized formula $\mathrm{MX}_{2}(\mathrm{M}=$ transition metal, $\mathrm{X}=$ chalcogen $(\mathrm{S}, \mathrm{Se}, \mathrm{Te}))$, with electronic properties resembling those of semiconducting, metallic and superconducting materials. $\mathrm{MoS}_{2}$ nanostructures from monolayers to bulk have been fabricated on a various number of rigid as well as flexible substrates by utilizing a variety of methods such as mechanical exfoliation, electrochemical exfoliation, thermal vapour sulphurisation of molybdenum or CVD of molybdenum oxide $\left(\mathrm{MoO}_{3}\right)$ and decomposition of thiomolybdates ${ }^{(23-28)}$.

In the presented work, we have synthesized two $\mathrm{MoS}_{2}$ thin film samples using Thermal Chemical Vapour Deposition. All the samples were then characterized using X-Ray Diffraction (XRD) method. Hence, the structural analysis of thermal CVD synthesized $\mathrm{MoS}_{2}$ nanofilms is presented in this report. The final aim is to judge how closely our results match to the standard values of structural parameters of $\mathrm{MoS}_{2}$, and thus comment on its usability in commercial applications.

\section{Materials and Methods}

In order to synthesize $\mathrm{MoS}_{2}$ thin films, commercially procured high purity molybdenum trioxide $(\mathrm{MoO} 3)(\mathrm{VI})$ powder and sulphur (S) flakes were used as precursors in a horizontal quartz tube furnace CVD system. A schematic diagram of the thermal Chemical Vapour Deposition apparatus for the growth of molybdenum disulphide is given in Figure 1 .

All the vessels, beakers and crucible used during the sample preparation underwent a standard cleaning procedure: prior to its insertion in the reaction chamber, the quartz tube was thoroughly cleansed with sulphuric acid and the substrate holder was washed with an organic solvent (acetone) and put under ultrasonic cleaning. The ultrasonic cleaning was performed in acetone for about 15-20 minutes to remove all the residual dirt from the material. $\mathrm{MoO}_{3}$ powder and $\mathrm{S}$ flakes were kept in separate ceramic crucibles separated by a distance of $15 \mathrm{~cm}$. Sulphur was placed upstream in the furnace and $\mathrm{MoO}_{3}$ downstream, in the central zone. Substrate was placed facing down towards the reactants. Reaction by-products were removed by the carrier gas, $\operatorname{argon}(\mathrm{Ar})$.

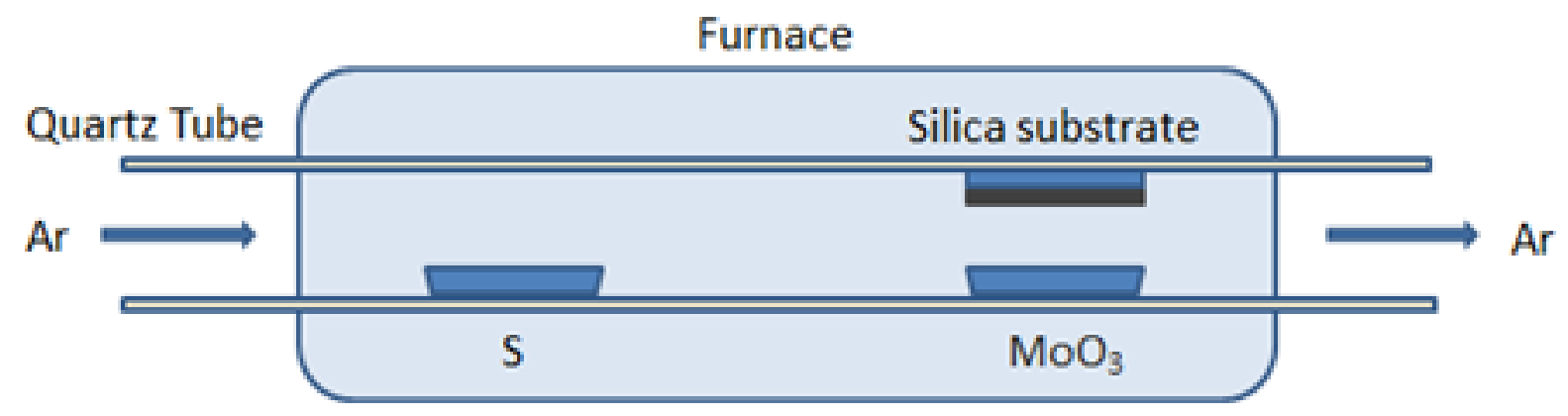

Fig 1. Schematic diagram of the CVD apparatus for growth of molybdenum disulphide

After purging the quartz tube with Ar gas for 15 minutes, the temperature of the quartz tube was raised to $700^{\circ} \mathrm{C}$ while keeping the flow rate through the tube at $200 \mathrm{sccm}$.

The tube was maintained at the mentioned temperature for 30 minutes to allow complete sulphurisation of $\mathrm{MoO}_{3}$ by thermal evaporation. Later, the CVD system was turned off keeping the flow rate constant, to allow the temperature of the quartz tube to come down to room temperature. This process took around 10 hours. After the completion the carrier gas flow was turned off and the crucibles were taken out. The crucible containing the substrate was found to have grown a thin film of $\mathrm{MoS}_{2}$ along with powder form of $\mathrm{MoS}_{2}$ during the reaction.

The $\mathrm{MoS}_{2}$ samples prepared by CVD have been characterized by X-ray Diffraction to determine information about their crystal structures. For this purpose, a commercial X-Ray diffractometer (model number D8 Advance, DAVINCI design) from Bruker has been employed. Equipped with a 60-90 position sample changer, it consumes $1600 \mathrm{~W}$ power for XRD measurements and is used in Bragg- Brentano parafocusing geometry. This results in both high resolution and high intensity of the diffracted beam. 


\section{Results and Discussions}

\subsection{X-Ray Diffractograms}

$\mathrm{X}$-ray Diffractograms were plotted, as shown in Figure 2, on the basis of XRD data to confirm the crystallinity, phase purity and structure of the synthesized samples of $\mathrm{MoS}_{2}$. In the plotted diffractograms, a range of angles $2 \theta$ with the most prominent peaks were selected. Gaussian line profiling was performed for each peak using Origin software and the full width at halfmaximum (FWHM) of the profile, area, and height values calculated for each Bragg line. This information was used to reveal the correlation between microstructural parameters of the prepared nanofilms and the standard values.

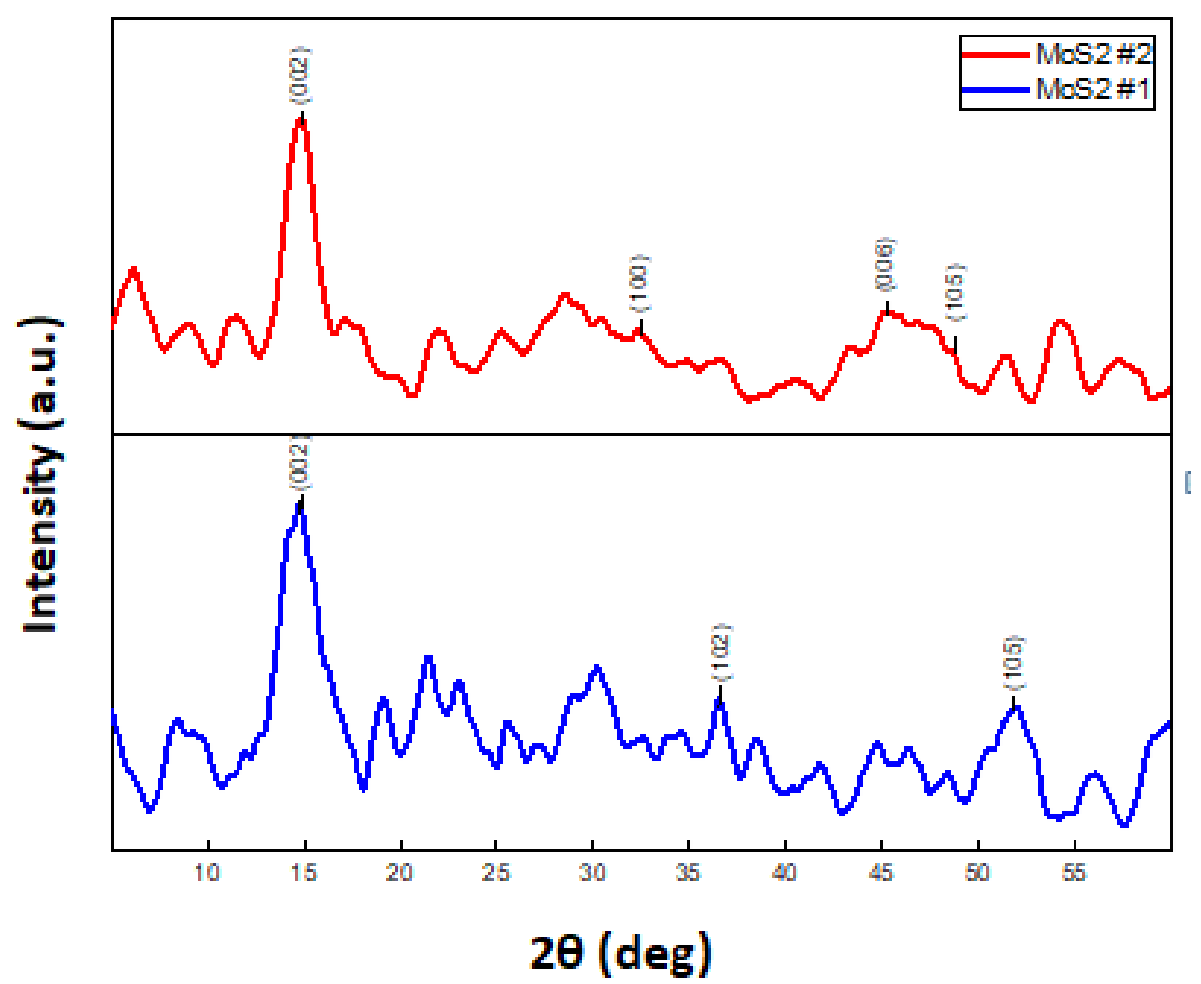

Fig 2. X-ray diffractograms of the $\mathrm{MoS}_{2}$ nanofilms samples

The major XRD peaks for the $\mathrm{MoS}_{2}$ samples are obtained at $14.76^{\circ}, 36.52^{\circ}$ and $51.88^{\circ}$ for our first sample and $14.84^{\circ}, 32.32^{\circ}$, $45.2^{\circ}$ and $48.56^{\circ}$ for the second sample. The positions of the major peaks for the $\mathrm{MoS}_{2}$ samples are most closely in agreement with the standard JCPDS data for $\mathrm{MoS}_{2}$. These peaks are attributed to hexagonal crystal structure with plane orientations represented in Table 1 .

Table 1. Calculated cell parameters, crystallite size, micro-strain and dislocation densities of the as-grown $\operatorname{mos}_{2}$ films

\begin{tabular}{|c|c|c|c|c|c|c|}
\hline $\mathrm{MoS}_{2}$ Sample & $\begin{array}{l}\text { Centre angle of peak } \\
(2 \theta)\end{array}$ & Plane (hkl) & $\mathrm{D}_{h k l}(\AA)$ & Crystallite size $(\AA)$ & $\begin{array}{l}\text { Microstrain } \\
\varepsilon\left(\mathrm{x} 10^{-2}\right)\end{array}$ & $\begin{array}{l}\text { Dislocation den- } \\
\text { sity }\left(10^{15} \mathrm{~m}^{-2}\right)\end{array}$ \\
\hline \multirow[t]{3}{*}{ MoS2\#1 } & 14.76 & 002 & 5.839 & 22.586 & 11 & 1.96 \\
\hline & 36.52 & 102 & 2.394 & 43.931 & 2.4 & 0.52 \\
\hline & 51.88 & 105 & 1.714 & 38.163 & 2 & 0.68 \\
\hline \multirow[t]{4}{*}{ MoS2\#2 } & 14.84 & 002 & 5.808 & 26.941 & 9 & 1.370 \\
\hline & 32.32 & 100 & 2.695 & 43.286 & 2.8 & 0.534 \\
\hline & 45.2 & 006 & 1.952 & 54.526 & 1.6 & 0.336 \\
\hline & 48.56 & 105 & 1.824 & 131.114 & 0.6 & 0.058 \\
\hline
\end{tabular}


The lattice spacings for the planes are calculated using Bragg's law:

$$
2 d \sin \theta=n \lambda
$$

For hexagonal structure, plane spacing $\mathrm{d}$ is related to lattice constants a, $\mathrm{c}$ and miller indices by the relation:

$$
\frac{1}{d_{h k l}^{2}}=\frac{4}{3}\left(\frac{h^{2}+h k+k^{2}}{a^{2}}\right)+\frac{l^{2}}{c^{2}}
$$

Combining with Bragg's law with this equation and rearranging with $\mathrm{n}=1$ approximation gives:

$$
\sin ^{2} \theta=\frac{\lambda^{2}}{4}\left[\frac{4}{3}\left(\frac{h^{2}+h k+k^{2}}{a^{2}}\right)+\frac{l^{2}}{c^{2}}\right]
$$

For hexagonal lattice cells, 'a' is calculated looking at planes of type hk0. Thus, (3) gives:

$$
a=\frac{\lambda}{\sqrt{3} \sin \theta}\left(h^{2}+h k+k^{2}\right)^{1 / 2}
$$

Similarly, 'c' can be calculated by looking for 001 type planes and substituting $\mathrm{h}=\mathrm{k}=0$ to obtain:

$$
c=\frac{\lambda l}{2 \sin \theta}
$$

The diffraction peak width contains microstructural information about the material as peak broadening is caused by nanocrystallite size and additionally, may also be a result of lattice strain and lattice defects. This broadening is quantified in terms of Full Width at Half Maximum (FWHM) and integral breadth depending upon the type of curve fitting. We have used FWHM values generated by our software for each peak and used them in the Scherrer formula to get the value of crystallite size, D:

$$
D_{h k l}=\frac{K \lambda}{\beta_{2 \theta} \cos \theta}
$$

Where, $\mathrm{K}$ is the Scherrer constant, $\beta$ is the diffraction peak width (in radians) and $\theta$ is the Bragg angle.

Microstrain or lattice strain is defined as the local deviation of d-spacings from the average value, which translates into variation in diffraction angles and results in diffraction peak broadening. The origin of these point defects may be interstitial or missing atoms, or substitution by different atoms; dislocations or twin boundary defects. The Williamson Hall method that we have used to calculate microstrain in this paper considers strain induced and crystallite size induced peak broadening as independent of each other. The diffraction line broadening being a result of strain induced as a result of crystal distortion and imperfection is calculated using the formula:

$$
\varepsilon=\frac{\beta}{4 \tan \theta}
$$

$\varepsilon$ Is the microstrain and $\beta$ is the broadening

Therefore, the strain-related diffraction peak broadening $\left(\beta_{\varepsilon}\right)$ is inversely proportional to $\tan \theta$, whereas peak broadening resulting from crystallite size $\left(\beta_{D}\right)$ varies as the inverse of $\cos \theta$. The total peak broadening is calculated as a sum of $\beta_{\varepsilon}$ and $\beta_{D}$ after accounting for the instrumental correction:

$$
\begin{gathered}
\beta_{h k l}=\beta_{D}+\beta_{\varepsilon} \\
\beta_{h k l}=\frac{K \lambda}{D \cos \theta}+4 \varepsilon \tan \theta
\end{gathered}
$$

Rearranging this equation gives the Williamson-Hall Equation:

$$
\beta_{h k l} \cos \theta=\frac{K \lambda}{D}+4_{\in} \sin \theta
$$


This equation results from a uniform deformation model that assumes that the crystal is isotropic in nature, i.e. crystallographic direction of measurement does not affect the value of strain.

For calculating values of lattice constants, plane (002) of the type (001) was used to find 'c' in both the samples. Then another plane (102) of the type (h0l) for the first sample, and (100) of the type (h00) for the second sample were used to find 'a' after substituting the obtained value of $\mathrm{c}$ into (3). These values are tabulated in Table 2.

Table 2. Calculated lattice parameters of the prepared samples

\begin{tabular}{lll}
\hline Sample Name & $\mathrm{a}(\AA)$ & $\mathrm{c}(\AA)$ \\
\hline $\mathrm{MoS}_{2} \# 1$ & 3.03 & 11.68 \\
$\mathrm{MoS}_{2} \# 2$ & 3.11 & 11.61 \\
\hline
\end{tabular}

The calculated parameters have been compared with the typical standardized values in Tables 3 and 4 , for validation of the results.

Table 3. Typical values of cell parameters of crystal structure of $\mathrm{MoS}_{2}$

\begin{tabular}{ll}
\hline $\mathrm{a}(\AA)$ & $\mathrm{b}(\AA)$ \\
\hline 3.158 & 12.221 \\
\hline
\end{tabular}

The major peaks obtained in our XRD pattern are reasonably consistent with the standardized peak values for $\mathrm{MoS}_{2}$ nanosheets. The calculated values of cell parameters of the crystal structure of $\operatorname{MoS}_{2}(\mathrm{a}=\mathrm{b}$ and $\mathrm{c})$ also agree with the typical values. The existence of a high intensity peak at $\left(\begin{array}{lll}0 & 0 & 2\end{array}\right)$ in both the samples implies periodicity in c-axis, which is indicative of multi-layer $\mathrm{MoS}_{2}$ nanosheets on $\mathrm{SiO}_{2}$ substrates.

Table 4. Typical values of central angle and miller indices obtained for $\mathrm{MoS}_{2}$ from XRD data

\begin{tabular}{lllll}
\hline \multirow{2}{*}{ S. No. } & $2 \theta$ (degrees) & \multicolumn{3}{c}{ Miller Indices } \\
\cline { 3 - 5 } & & $\mathrm{h}$ & $\mathrm{k}$ & $\mathrm{l}$ \\
\hline 1 & 14.475 & 0 & 0 & 2 \\
2 & 32.708 & 1 & 0 & 0 \\
3 & 35.937 & 1 & 0 & 2 \\
4 & 44.433 & 0 & 0 & 6 \\
5 & 49.997 & 1 & 0 & 5 \\
\hline
\end{tabular}

\subsection{Applications}

$\mathrm{MoS}_{2}$ finds application in a diverse range of fields due to its novel and desirable electronic properties and chemical structure. It has been shown that multilayer plasma-treated $\mathrm{MoS}_{2}$ transistors can act as affordable, highly durable, non-volatile memory devices. Moreover, the combination of two-dimensional materials like $\mathrm{MoS}_{2}$ and graphene with high- $\kappa$ dielectric materials can lead to the fabrication of non-volatile memory devices that possess increased memory window as well as stable retention, while also allowing multibit information storage ${ }^{(29,30)}$. A film composed of the combination of graphene oxide (GO) nanosheets with 2-D $\mathrm{MoS}_{2}$ has been used to synthesize memory devices.

The $\mathrm{MoS}_{2}$ enhances the film conductivity, facilitating migration of oxygen in GO. This device has displayed non-volatile, rewritable, bistable switching with low voltage and an optimally high value of ON/OFF current ratio. Another $\mathrm{MoS}_{2}$-graphene composite has shown superior electrochemical performance, which can serve as an application for anode material in lithium-ion batteries. This composite consisted of a $\mathrm{MoS}_{2}$-coated 3D network of graphene and also showed superior high-current-density performance.

This composite also has applications in other areas of clean energy ${ }^{(31,32)}$. Owing to its unique structure and superior mechanical, biological and physicochemical properties, $\mathrm{MoS}_{2}$ nanosheets can be used for various environmental applications related to water, such as membrane-based separation, contaminant absorption, or as disinfectants for biomedical devices, utilizing their antibacterial properties in absence of light ${ }^{(33)}$. Combining $\mathrm{MoS}_{2}$ with other semiconductor materials to alter its bulk-to-single layer structure can enhance the photocatalytic performance. Different types of $\mathrm{MoS}_{2}$ nanoparticles based on their structural components have been studied for their properties and applications in photocatalyst systems ${ }^{(8,34)}$. 
Ultrasmall nanodots, complex nanostructures and printed 3-D nanoarchitecture forms of $\mathrm{MoS}_{2}$ have been shown to be effective for photothermal therapy due to their desirable properties such as low cytotoxicity and stability. Using fluorescent quantum dots and arginine-glycine-aspartic (RGD) containing peptides has been used to produce functionalized single-layered $\mathrm{MoS}_{2}$ nanosheets that have shown remarkable properties of fluorescence, photothermal conversion and cancer-targeting. $\mathrm{MoS}_{2}$-PEG can be used for fast targeted drug delivery as it shows minimal toxicity to cells ${ }^{(35)}$. Nanoparticle forms of $\mathrm{MoS}_{2}$ have also shown promising behaviour as potential tools for treatment of cancer and Alzheimer's, with varying amounts of toxicities in different Mo based nanoforms ${ }^{(36)}$.

Mechanical flexibility of $\mathrm{MoS}_{2}$ also makes it desirable for use in flexible electronics. It has been shown that mono and multi-layer $\mathrm{MoS}_{2}$ can be used in conventional and tunnel field effect transistors, owing to their direct and indirect band-gap respectively. The effect of varying sulfurization temperature on the annealing of $\mathrm{MoO}_{3}$ film has been studied in detail to obtain $2 \mathrm{D} \mathrm{MoS}_{2}$ films spanning a large area for nanoelectronic applications such as ferroelectric FETs (FeFET) memory ${ }^{(37)}$. Utilizing the optical properties of $\mathrm{MoS}_{2}$ appropriately, an $\mathrm{MoS}_{2}$ based photodetector has the ability to detect photons in the visible range ${ }^{(38)}$. $\mathrm{MoS}_{2}$ based optoelectronic devices, when irradiated with UV - visible light, display useful properties for application in room-temperature optoelectronic $\mathrm{NO}_{2}$ sensors. $\mathrm{MoS}_{2}$ enriched with sulphur-vacancies, upon functionalization with $\mathrm{ZnO}$ quantum dots, showed superior performance, stability, fast response rate, reliable selectivity, full reversibility as well as subppb detection to $\mathrm{NO}_{2}$ and humidity resistance at room temperature ${ }^{(39)}$. Moreover, apart from finding use in $\mathrm{NO}_{2}$ sensors, a nanocomposite film incorporating indium oxide $\left(\operatorname{In}_{2} \mathrm{O}_{3}\right)$ and $\mathrm{MoS}_{2}$, in a nanccube or flower-like layer-by-layer synthesized structure, has found specific applications as a formaldehyde vapour sensor at room temperature ${ }^{(40)}$.

\section{Conclusions}

Molybdenum disulphide $\left(\mathrm{MoS}_{2}\right)$ nanofilms are successfully synthesized on silicon dioxide substrates by the thermal Chemical Vapour Deposition (CVD) technique, using molybdenum trioxide $\left(\mathrm{MoO}_{3}\right)(\mathrm{VI})$ powder and sulphur (S) flakes as precursors. Then, the CVD grown $\mathrm{MoS}_{2}$ samples are characterized using X-ray Diffraction method in order to study the structural analysis of the prepared samples. As the obtained XRD-peaks and lattice parameters of the $\mathrm{MoS}_{2}$ nanosheets are in accordance with the standardized values, and the calculated values of cell parameters of the crystal structure of $\operatorname{MoS}_{2}(a=b$ and $c)$ also agree with the typical values, the sheets have been grown properly via CVD mechanism and characterization through XRD provides information about the grown sample. The existence of a high intensity peak at $\left(\begin{array}{ll}0 & 0\end{array}\right)$ in both the samples implies periodicity in c-axis, which is indicative of multi-layer $\mathrm{MoS}_{2}$ nanosheets on $\mathrm{SiO}_{2}$ substrates. This report also calculates the microstrain and dislocation density to provide an insight into the defects present in the grown sample.

Research interest towards incorporating $\mathrm{MoS}_{2}$ owing to its structure and properties has been steadily mounting since the past few years due to its novel applications as hybrid structures in next-generation devices. A few of these applications have been discussed in brief, each of which can be greatly benefitted by the wide range of benefits offered by $\mathrm{MoS}_{2}$ structures. While the future scope of applications of $\mathrm{MoS}_{2}$ is extensively varied, the authors of this paper intend to specifically work on incorporating $\mathrm{MoS}_{2}$ in memory devices as well as solar cells for obtaining novel benefits in the field of energy.

\section{Acknowledgment}

The authors would like to extend the deepest gratitude to the Department of Applied Physics, Delhi Technological University for giving the opportunity to work on this topic. The authors would also like to thank the peers for their constant support and encouragement.

\section{References}

1) Mak KF, Shan J. Photonics and optoelectronics of 2D semiconductor transition metal dichalcogenides. Nature Photonics. 2016;10(4):216-226. Available from: https://dx.doi.org/10.1038/nphoton.2015.282.

2) Mak KF, Lee C, Hone J, Shan J, Heinz TF. Atomically ThinMoS2: A New Direct-Gap Semiconductor. Physical Review Letters. 2010;105(13):136805-136805. Available from: https://dx.doi.org/10.1103/physrevlett.105.136805.

3) Zhang X, Zhang S, Xie Y, Huang J, Wang L, Cui Y, et al. Tailoring the nonlinear optical performance of two-dimensional MoS2 nanofilms via defect engineering. Nanoscale. 2018;10(37):17924-17932. Available from: https://doi.org/10.1039/C8NR05653F.

4) Islam MA, Church J, Han C, Chung HS, Ji E, Kim J, et al. Noble metal-coated MoS2 nanofilms with vertically-aligned 2D layers for visible light-driven photocatalytic degradation of emerging water contaminants. Scientific Reports. 2017;7. Available from: https://doi.org/10.1038/s41598-017-14816-9.

5) Stern C, Grinvald S, Kirshner M, Sinai O, Oksman M, Alon H, et al. Growth Mechanisms and Electronic Properties of Vertically Aligned MoS2. Scientific Reports. 2018;8(1):16480-16480. Available from: https://dx.doi.org/10.1038/s41598-018-34222-z.

6) Son D, Chae S, Kim M, Choi MK, Yang J, Park K, et al. Colloidal synthesis of uniform-sized molybdenum disulfide nanosheets for wafer-scale flexible nonvolatile memory. Advanced Materials. 2016;28. Available from: https://doi.org/10.1002/adma.201602391. 
7) Shinde SM, Kalita G, Tanemura M. Fabrication of poly(methyl methacrylate)-MoS2/graphene heterostructure for memory device application. Journal of Applied Physics. 2014;116(21):214306-214306. Available from: https://dx.doi.org/10.1063/1.4903552.

8) Wang Z, Mi B. Environmental applications of 2D molybdenum disulfide (MoS2) nanosheets. Environmental Science \& Technology. 2017;51(15):8229-8244. Available from: https://doi.org/10.1021/acs.est.7b01466.

9) Liu T, Liu Z. 2D MoS2 nanostructures for biomedical applications. Advanced Healthcare Materials. 2018;7(8):1701158-1701158. Available from: https://doi.org/10.1002/adhm.201701158.

10) Lopez-Sanchez O, Lembke D, Kayci M, Radenovic A, Kis A. Ultrasensitive photodetectors based on monolayer MoS2. Nature Nanotechnology. 2013;8(7):497-501. Available from: https://dx.doi.org/10.1038/nnano.2013.100.

11) Pu J, Yomogida Y, Liu KK, Li LJ, Iwasa Y, Takenobu T. Highly Flexible MoS2 Thin-Film Transistors with Ion Gel Dielectrics. Nano Letters. 2012;12(8):40134017. Available from: https://dx.doi.org/10.1021/nl301335q.

12) Zhang Y, Tan YW, Stormer HL, Kim P. Experimental observation of the quantum Hall effect and Berry's phase in graphene. Nature. 2005;438(7065):201204. Available from: https://dx.doi.org/10.1038/nature04235.

13) Balandin AA, Ghosh S, Bao W, Calizo I, Teweldebrhan D, Miao F, et al. Superior Thermal Conductivity of Single-Layer Graphene. Nano Letters. 2008;8(3):902-907. Available from: https://dx.doi.org/10.1021/nl0731872.

14) Bolotin KI, Sikes KJ, Jiang Z, Klima M, Fudenberg G, Hone J, et al. Ultrahigh electron mobility in suspended graphene. Solid State Communications. 2008;146(9-10):351-355. Available from: https://dx.doi.org/10.1016/j.ssc.2008.02.024.

15) Lee C, Wei X, Kysar JW, Hone J. Measurement of the Elastic Properties and Intrinsic Strength of Monolayer Graphene. Science. 2008;321(5887):385-388. Available from: https://dx.doi.org/10.1126/science.1157996.

16) Sun Z, Dong N, Xie K, Xia W, König D, Nagaiah TC, et al. Nanostructured Few-Layer Graphene with Superior Optical Limiting Properties Fabricated by a Catalytic Steam Etching Process. The Journal of Physical Chemistry C. 2013;117(22):11811-11817. Available from: https://dx.doi.org/10.1021/jp401736n.

17) Kim K, Lara-Avila S, He H, Kang H, Park Y, Yakimova R, et al. Thermal Stability of Epitaxial Graphene Electrodes for Conductive Polymer Nanofiber Devices. Crystals. 2017;7(12):378-378. Available from: https://dx.doi.org/10.3390/cryst7120378.

18) Geim AK, Novoselov KS. The rise of graphene. Nature Materials. 2007;6(3):183-191. Available from: https://dx.doi.org/10.1038/nmat1849.

19) Reina G, González-Domínguez JM, Criado A, Vázquez E, Bianco A, Prato M. Promises, facts and challenges for graphene in biomedical applications. Chemical Society Reviews. 2017;46(15):4400-4416. Available from: https://dx.doi.org/10.1039/c7cs00363c.

20) Kuhn KJ. Considerations for Ultimate CMOS Scaling. IEEE Transactions on Electron Devices. 2012;59(7):1813-1828. Available from: https://dx.doi.org/ 10.1109/ted.2012.2193129.

21) Schwierz F. Graphene transistors. Nature Nanotechnology. 2010;5(7):487-496. Available from: https://dx.doi.org/10.1038/nnano.2010.89.

22) Schwierz F. Graphene Transistors: Status, Prospects, and Problems. Proceedings of the IEEE. 2013;101(7):1567-1584. Available from: https://dx.doi.org/ 10.1109/jproc.2013.2257633.

23) Sun J, Li X, Guo W, Zhao M, Fan X, Dong Y, et al. Synthesis methods of two-dimensional MoS2: a brief review. Crystals. 2017;7(7). Available from: https://doi.org/10.3390/cryst7070198.

24) Deokar G, Vignaud D, Arenal R, Louette P, Colomer JF. Synthesis and characterization of MoS2nanosheets. Nanotechnology. 2016;27(7):075604-075604. Available from: https://dx.doi.org/10.1088/0957-4484/27/7/075604.

25) Liu KK, Zhang W, Lee YH, Lin YC, Chang MT, Su CY, et al. Growth of large-area and highly crystalline MoS2 thin layers on insulating substrates. Nano Letters. 2012;12(3):1538-1582. Available from: https://doi.org/10.1021/nl2043612.

26) Wang H, Skeldon P, Thompson G, Wood GC. Synthesis and characterization of molybdenum disulphide formed from ammonium tetrathiomolybdate. Journal of Materials Science. 1997;32:497-502. Available from: https://doi.org/10.1023/a:1018538424373.

27) Liu N, Kim P, Kim JH, Ye JH, Kim S, Lee CJ. Large-area atomically thin MoS2 nanosheets prepared using electrochemical exfoliation. ACS Nano. 2014;8(7):6902-6910. Available from: https://doi.org/10.1021/nn5016242.

28) Magda GZ, Pető J, Dobrik G, Hwang C, Biró LP, Tapasztó L. Exfoliation of large-area transition metal chalcogenide single layers. Scientific Reports. 2015;5(1):14714-14714. Available from: https://dx.doi.org/10.1038/srep14714.

29) Chen M, Nam H, Wi S, Priessnitz G, Gunawan IM, Liang X. Multibit Data Storage States Formed in Plasma-Treated MoS2 Transistors. ACS Nano. 2014;8(4):4023-4032. Available from: https://dx.doi.org/10.1021/nn501181t.

30) Zhang E, Wang W, Zhang C, Jin Y, Zhu G, Sun Q, et al. Tunable charge-trap memory based on few-layer MoS2. ACS Nano. 2015;9(1):612-619. Available from: https://doi.org/10.1021/nn5059419.

31) Yin Z, Zeng Z, Liu J, He Q, Chen P, Zhang H. Memory devices using a mixture of MoS2 and graphene oxide as the active layer. Small. 2013;9(5):727-731. Available from: https://doi.org/10.1002/smll.201201940.

32) Cao X, Shi Y, Shi W, Rui X, Yan Q, Kong J, et al. Preparation of MoS 2-coated three-dimensional graphene networks for high-performance anode material in lithium-ion batteries. Small. 2013;9(20):3433-3438. Available from: https://doi.org/10.1002/smll.201202697.

33) Tang K, Wang L, Geng H, Qiu J, Cao H, Liu X. Molybdenum disulfide (MoS2) nanosheets vertically coated on titanium for disinfection in the dark". Arabian Journal of Chemistry;13(1):1612-1623. Available from: https://doi.org/10.1016/j.arabjc.2017.12.013.

34) Vattikuti SVP, Byon C. Molybdenum disulfide-based photocatalysis: bulk-to-single layer structure and related photomechansim for environmental applications. In: and others, editor. Nanoscaled Films and Layers. IntechOpen. 2017.

35) Bazaka K, Levchenko I, Lim JWM, Baranov O, Corbella C, Xu S, et al. MoS2-based nanostructures: synthesis and applications in medicine. Journal of Physics D: Applied Physics. 2019;52(18):183001-183001. Available from: https://dx.doi.org/10.1088/1361-6463/ab03b3.

36) Sobanska Z, Zapor L, Szparaga M, Stepnik M. Biological effects of molybdenum compounds in nanosized forms under $<\mathrm{i}>$ in vitro $</ \mathrm{i}>$ and $<\mathrm{i}>\mathrm{in}$ vivo $</ \mathrm{i}>$ conditions. International Journal of Occupational Medicine and Environmental Health. 2020;33(1):1-19. Available from: https://dx.doi.org/10.13075/ ijomeh.1896.01411.

37) Romanov RI, Kozodaev MG, Myakota DI, Chernikova AG, Novikov SM, Volkov VS, et al. Synthesis of Large Area Two-Dimensional MoS2 Films by Sulfurization of Atomic Layer Deposited MoO3 Thin Film for Nanoelectronic Applications. ACS Applied Nano Materials. 2019;2(12):7521-7531. Available from: https://dx.doi.org/10.1021/acsanm.9b01539.

38) Ullah MS, Yousuf AHB, Es-Sakhi AD, Chowdhury MH. Analysis of optical and electronic properties of MoS2 for optoelectronics and FET applications. AIP Conference Proceedings. 2018;1957(1). Available from: https://doi.org/10.1063/1.5034320.

39) Xia Y, Hu C, Guo S, Zhang L, Wang M, Peng J, et al. Sulfur-Vacancy-Enriched MoS2 Nanosheets Based Heterostructures for Near-Infrared Optoelectronic NO2 Sensing. ACS Applied Nano Materials. 2020;3(1):665-673. Available from: https://dx.doi.org/10.1021/acsanm.9b02180. 
40) Zhang D, Jiang $\mathrm{C}$, Wu J. Layer-by-layer assembled In2O3 nanocubes/flower-like MoS2 nanofilm for room temperature formaldehyde sensing. Sensors and Actuators B: Chemical. 2018;273:176-184. Available from: https://doi.org/10.1016/j.snb.2018.06.044. 